\title{
Scinderin suppresses cell proliferation and predicts the poor prognosis of hepatocellular carcinoma
}

\author{
BIN ZHOU $^{1 *}$, TIAN-WEI CHEN ${ }^{2 *}$, YA-BO JIANG $^{1 *}$, XU-BIAO WEI $^{1}$, \\ CHONG-DE LU ${ }^{1}$, JING-JING LI' ${ }^{2}$, DONG XIE ${ }^{2}$ and SHU-QUN CHENG ${ }^{1}$ \\ ${ }^{1}$ Department of Hepatic Surgery VI, Eastern Hepatobiliary Surgery Hospital, Second Military Medical University, \\ Shanghai 200438; ${ }^{2}$ Laboratory of Molecular Oncology, Institute for Nutritional Sciences, Shanghai Institutes \\ for Biological Sciences, Chinese Academy of Sciences, Shanghai 200031, P.R. China
}

Received March 19, 2019; Accepted November 26, 2019

DOI: $10.3892 / \mathrm{ol} .2020 .11262$

\begin{abstract}
Hepatocellular carcinoma (HCC) remains an intractable disease despite numerous advancements made in the available treatments over recent decades. Therefore, investigation of the underlying pathogenesis of HCC is urgently required. Our previous microarray result showed that SCIN was generally downregulated in 23 paired tumor/normal tissues. Reverse transcription-quantitative PCR, western blotting and immunohistochemistry were performed in the present study in order to detect the expression of scinderin (SCIN). Lentivirus-mediated gene delivery was used in order to produce SCIN-manipulated cell lines. MTT and crystal violet assays were performed in order to investigate cell growth, and fluorescence-activated cell sorting analysis was used in order to determine cell cycle distribution. SCIN was downregulated in HCC samples, and low SCIN expression predicted the poor prognosis of patients with HCC. Notably, SCIN may have the potential to serve as an independent risk factor for overall survival (3-year overall survival rate of 28.6 and $10.3 \%$ in high SCIN expression and low SCIN expression groups, respectively) and disease-free survival (3-year recurrence rate of 71.4 and $84.6 \%$ in high SCIN expression and low SCIN expression groups, respectively) in HCC. SCIN inhibited HCC cell proliferation both in vitro and in subcutaneous tumor formation assay. Furthermore, SCIN decreased the levels of phosphorylated STAT3, thereby downregulating cyclin A1 levels in HCC cells. The results of the present study
\end{abstract}

Correspondence to: Dr Shu-Qun Cheng, Department of Hepatic Surgery VI, Eastern Hepatobiliary Surgery Hospital, Second Military Medical University, 225 Changhai Road, Shanghai 200438, P.R. China

E-mail: chengshuqun@aliyun.com

*Contributed equally

Key words: scinderin, hepatocellular carcinoma, prognosis, proliferation, STAT3 demonstrate the tumor suppressive role of SCIN in HCC, providing a candidate strategy to treat this disease.

\section{Introduction}

Hepatocellular carcinoma (HCC) is the third most frequent cause of cancer-associated mortality worldwide (1). There is a high incidence in East and South East Asia, which is associated with the prevalence of hepatitis B virus in these regions (2). Surgical resection remains the principle choice of treatment for patients with preserved liver function; however, $60-70 \%$ of patients develop metastasis and recurrence within 5 years of surgery or transcatheter arterial chemoembolization (3). Although several clinicopathological features, including poor differentiation status, large-sized tumors and portal venous invasion, have been demonstrated to contribute to the poor prognosis of patients with HCC prior to surgery (4), the molecular mechanisms underlying the development of HCC remain unknown. Thus, it is critical to uncover the pathogenesis of HCC.

Scinderin (SCIN) is a calcium-dependent actin filament serving and capping protein that belongs to the gesolin superfamily (5). Previous studies have demonstrated that SCIN regulates vesicle transport and exocytosis in endocrine and secretory cells by organizing the cytoskeleton $(6,7)$. SCIN can also regulate cell differentiation through the MAP kinases P38 and ERK1/2-mediated signaling pathways $(8,9)$. The dysregulation of SCIN has been reported in several types of cancer, but these reports have not been consistent. In lung and prostate cancer cell lines, SCIN-knockdown was demonstrated to inhibit cell proliferation $(10,11)$, and SCIN was overexpressed in gastric cancer (12). SCIN-knockdown in gastric cell lines decreased the metastatic ability of these cells (13). These results suggest that SCIN may function as an oncogene. However, in megakaryoblastic leukemia and acute myeloid leukemia, SCIN was downregulated, and overexpression of SCIN in leukemia cell lines was demonstrated to inhibit cell proliferation $(8,14)$. Furthermore, SCIN was demonstrated to be weakly expressed in head and neck cancer, and its expression could not predict prognosis (15). Thus, the function of SCIN in cancer remains under debate.

The present study examined the expression pattern of SCIN in HCC and investigated the exact function of this 
protein in HCC cells. Key molecules influenced by SCIN were also detected.

\section{Materials and methods}

Patients and specimens. Fresh tumor tissue samples and paired non-cancerous liver tissue samples of 12 patients with HCC were obtained following hepatectomy at the Eastern Hepatobiliary Hospital (Shanghai, China) between March 2016 and May 2016. A total of 60 paraffin-embedded HCC samples were also collected from the Eastern Hepatobiliary Hospital, between March 2005 and August 2007. Patients were aged between 29 and 70 years (mean age, 48 years). There were 51 men $(85 \%)$ and 9 women $(15 \%)$. The key inclusion criteria were as follows: HCC diagnosed by biopsy or by the non-invasive criteria of the European Association for the Study of Liver guidelines (16). The key exclusion criteria included patients with a history of other malignancies in the past 5 years. A tissue microarray (TMA) was created from these samples. Western blot assays were performed in order to confirm the specificity of SCIN staining in the fresh HCC tissues and paired non-cancerous liver tissues, and in the SK-HEP-1 and YY-8103 cell lines. The patients had not received radiotherapy or chemotherapy prior to surgical treatment. The clinical and pathological data of the 60 patients with HCC were collected. Clinical follow-up information was obtained by telephone or from the outpatient records.

The present study was approved by the Medical Ethics Committee of Eastern Hepatobiliary Surgery Hospital and all patients provided written informed consent prior to the study. Participants were recruited and experiments were conducted in the Eastern Hepatobiliary Surgery Hospital.

\section{Cell culture conditions}

Cell culture. The HCC cell lines YY-8103, SK-HEP-1, QGY, MHCC97-H, Huh7 and 293T were purchased from The Cell Bank of Type Culture Collection of the Chinese Academy of Sciences. CSQT-2 was established in our laboratory as previously described (17). The cells were maintained in DMEM (Gibco; GE Healthcare Life Sciences) supplemented with $10 \%$ fetal bovine serum (ExCell Bio), penicillin $(100 \mathrm{U} / \mathrm{ml})$ and streptomycin $(100 \mathrm{U} / \mathrm{ml})$ at $37^{\circ} \mathrm{C}$ in a humidified $5 \% \mathrm{CO}_{2}$ atmosphere.

Western blot analysis. Total protein was extracted from SK-HEP-1 and YY-8103 cells and HCC tissue samples using RIPA buffer with protease inhibitor (Sigma-Aldrich; Merck $\mathrm{KGaA}$ ), and protein concentrations were determined via the Bradford method. Proteins $(30 \mu \mathrm{g})$ were separated by $10 \%$ SDS-PAGE, and subsequently transferred onto polyvinylidene difluoride membranes (EMD Millipore). The membranes were blocked for $1 \mathrm{~h}$ at room temperature with $5 \%$ non-fat milk in TBST solution, prior to incubation with the primary antibody anti-SCIN (1:1,000; cat. no. HPA024264; Sigma-Aldrich; Merck KGaA), anti-cyclin A1 (1:1,000; cat. no. 556600; BD Pharmingen; BD Biosciences), anti-STAT3 and anti-pSTAT3 (Tyr705) (1:1,000; cat. nos. 4904S and 9145S, respectively; Cell Signaling Technology, Inc.) overnight at $4^{\circ} \mathrm{C}$. Membranes were washed three times with TBST, and were incubated with the horseradish peroxidase-conjugated secondary antibodies anti-rabbit IgG and anti-mouse $\operatorname{IgG}(1: 1,000$; cat. nos. 7074 and 7076, respectively; Cell Signaling Technology, Inc.) for $1 \mathrm{~h}$ at room temperature. Membranes were re-washed with TBST three times, and protein bands were subsequently visualized using an enhanced chemiluminescence system (cat. no. 32209, Pierce ${ }^{\mathrm{TM}}$ ECL; Thermo Fisher Scientific, Inc.), according to the manufacturer's protocol (18). Protein expression was quantified using ImageJ 1.8 software (National Institutes of Health), and the number under each band was calculated as the result divided by GAPDH.

Vector construction and transfection. The pHAGE-EF1aIRES-GFP vector, which was a gift from Chenqi $\mathrm{Xu}$ at the Shanghai Institute of Biochemistry and Cell Biology, CAS, was used in order to generate the overexpression virus in 293T cells. The SCIN short hairpin (sh)-RNA plasmid was purchased from GeneChem Biotechnology. Fluorescence-activated cell sorting (FACS) was used in order to enrich green fluorescent protein (GFP)-positive cells.

In order to establish stable cell lines, the pHAGE-EF1aIRES-GFP empty vector was used as the control for SCIN overexpression and a plasmid containing a scrambled sequence (5'-CCTAAGGTTAAGTCGCCCTCGCTC GAGCGAGGGCGACTTAACCTTAGG-3'; cat. no. 1864; Addgene) was used as the control for SCIN-knockdown. In order to produce the lentivirus, 293T cells were transfected with the aforementioned core plasmids, along with the packaging plasmids psPAX2 and pMD2.G (cat. nos. 12260 and 12259, respectively; Addgene) at a ratio of 12:8:4 $\mu \mathrm{g}$ and the Lipofectamine ${ }^{\circledR} 2000$ transfection reagent (Thermo Fisher Scientific, Inc.). Viruses were harvested at $72 \mathrm{~h}$ post transfection (medium was refreshed every $24 \mathrm{~h}$ ). Transfection of HCC cells was performed in 6-well plates with polybrene at a final concentration of $2.5 \mu \mathrm{g} / \mathrm{ml}$ for $24 \mathrm{~h}$. Cells were sorted for GFP signals by FACS using FACSAria (Beckman Coulter, Inc.). Overexpression and knockdown efficacies were demonstrated by western blot analysis.

Reverse transcription-quantitative PCR (RT-qPCR) analysis (19). Total RNA from tissues was extracted using TRIzol $^{\circledR}$ reagent (Invitrogen; Thermo Fisher Scientific, Inc.), according to the manufacturer's protocol. Total RNA was reverse transcribed to cDNA (cat. no. A3500; Promega Corporation). qPCR was subsequently performed using the CFX96 Real-Time System (Bio-Rad Laboratories, Inc.), and the SYBR ${ }^{\circledR}$ Green 26 Master mix (Invitrogen; Thermo Fisher Scientific, Inc.) was used in a total reaction volume of $10 \mu \mathrm{l}$. The PCR procedure was as follows: $95^{\circ} \mathrm{C}$ for $7 \mathrm{~min} ; 95^{\circ} \mathrm{C}$ for $5 \mathrm{sec}$, $60^{\circ} \mathrm{C}$ for $30 \mathrm{sec}$ (data collection), $60^{\circ} \mathrm{C}$ for $30 \mathrm{sec}, 40$ cycles; $60-95^{\circ} \mathrm{C}$ (data collection), $20^{\circ} \mathrm{C}$ for $10 \mathrm{sec}$. The primers used were as follows: SCIN forward, 5'-CGAGGCTTCACCTAC CA-3' and reverse, 5'-CACTCTACGACCCTTCACA-3'; and actin (reference gene) forward, 5'-GATCATTGCTCCTCC TGAGC-3' and reverse, 5'-ACTCCTGCTTGCTGATCCAC-3'.

Immunohistochemistry (IHC). IHC staining was performed as previously described (20). All sections (including TMA) were deparaffinized with xylene at $65^{\circ} \mathrm{C}$ for $30 \mathrm{~min}$ and at room temperature for $20 \mathrm{~min}$, and rehydrated in a descending ethanol series. Subsequently, the $10-\mu \mathrm{m}$ sections were submerged in 
EDTA antigenic retrieval buffer ( $\mathrm{pH}$ 8.0) and microwaved in boiling water for $20 \mathrm{~min}$ for antigenic retrieval. Tissue sections were incubated with $0.3 \% \mathrm{H}_{2} \mathrm{O}_{2}$ at room temperature for $15 \mathrm{~min}$ to inhibit endogenous peroxidase activity and subsequently blocked with $1 \%$ normal goat serum (cat. no. 5425; Cell Signaling Technology, Inc.) at room temperature for $30 \mathrm{~min}$ in order to decrease non-specific binding. Tissue sections were incubated with rabbit polyclonal anti-SCIN antibody (1:200; cat. no. HPA024264; Sigma-Aldrich; Merck KGaA) overnight at $4^{\circ} \mathrm{C}$. Following three washes with TBS, sections were incubated with horseradish peroxidase-linked anti-rabbit antibody (1:200; cat. no. 7074; Cell Signaling Technology, Inc.) for $30 \mathrm{~min}$ at $37^{\circ} \mathrm{C}$. The slides were subsequently stained with 3,3'-diaminobenzidine. Images were captured at $\mathrm{x} 40$ and x200 magnification using a light microscope (BX61; Olympus Corporation).

The stained tissue sections were scored independently by two pathologists blinded to the clinical parameters, and the final score was the average of the scores recorded by the two observers. The present study used the intensity and extent of staining in order to evaluate SCIN expression. The staining intensity was scored as follows: 0 , no staining; 1, weak staining exhibited as light yellow; 2 , moderate staining exhibited as yellow/brown; and 3, strong staining exhibited as brown. The extent of staining was scored according to the percentages of the positively stained areas as follows: 0,$0 ; 1,1-25 ; 2,26-50 ; 3,51-75$; and $4,76-100 \%$. The product of the intensity score multiplied by the extent score was used as the final staining H-score (0-12) for SCIN. For the purpose of statistical evaluation, tumors with an $\mathrm{H}$-score of $<6$ were classified into the low SCIN expression group and those with an $\mathrm{H}$-score of $\geq 6$ were classified into the high SCIN expression group.

Colony formation assay. For the colony formation assay, SK-HEP-1 and YY-8103 cells were seeded into 6-well plates at a density of 1,000 cells/well and cultured for 7 days. Subsequently, the cells were fixed with $10 \%$ methanol at room temperature for $10 \mathrm{~min}$ and stained with $1 \%$ crystal violet at room temperature for $1 \mathrm{~min}$. The experiment was performed in triplicate for each group of cells. Images were captured at x40 and x100 magnification using an inverted light microscope (IX71; Olympus Corporation).

MTT assay. SK-HEP-1 and YY-8103 cells were seeded into 96-well plates at a density of 500 cells/well. Every $24 \mathrm{~h}$ of the subsequent 5 days, cells were incubated with $0.2 \%$ MTT solution (cat. no. A600799; Sangon Biotech Co. Ltd.) for $4 \mathrm{~h}$ at $37^{\circ} \mathrm{C}$. Following the MTT incubation, the purple formazan crystals were dissolved in $200 \mu \mathrm{l}$ of DMSO (cat. no. A100231; Sangon Biotech Co., Ltd.) and cell proliferation was subsequently analyzed at a wavelength of $490 \mathrm{~nm}$.

Cell cycle analysis. Cell cycle progression was analyzed by flow cytometry. SK-HEP-1 and YY-8103 cells at $~ 70 \%$ confluence were washed three times with PBS and fixed with $75 \%$ ethanol at $4^{\circ} \mathrm{C}$ overnight. Subsequently, the cells were stained with propidium iodide and RNase overnight at $4^{\circ} \mathrm{C}$. Samples were analyzed using the Cell Lab Quanta Flow Cytometer (Beckman Coulter, Inc.).
Tumor formation in an animal model. An equal number of SK-HEP-1 and YY-8103 cells ( $1 \times 10^{6}$ cells) were injected subcutaneously into the left and right flanks of nine male, 5-week old, nude mice (weight, 15-17.5 g; Shanghai SLAC Laboratory Animal Co., Ltd.), respectively. Mice were housed with free access to regular chow diet under specific pathogen-free conditions in laboratory cages at $23 \pm 3^{\circ} \mathrm{C}$ at $35 \pm 5 \%$ humidity under a 12-h dark/light cycle. The tumorigenesis procedure was recorded by measuring solid tumors in 3 dimensions with a caliper for 4 weeks. Animals were sacrificed 4 weeks after the injection. The maximum tumor volume presented was $103.68 \mathrm{~mm}^{3}$. The experiments on mice were approved by the Ethics Committee of Eastern Hepatobiliary Surgery Hospital.

Statistical analysis. Statistical analyses were performed using SPSS software (version 19.0; IBM Corp.). The significance of SCIN mRNA levels was determined using Student's t-test. The $\chi^{2}$ test was used in order to analyze the association between SCIN expression and clinicopathological characteristics. Survival times were evaluated using Kaplan-Meier survival curves, and differences in survival were analyzed using the log-rank test. The significance of variables for survival was analyzed by multivariate survival analysis using Cox's regression model. $\mathrm{P}<0.05$ was considered to indicate a statistically significant difference.

\section{Results}

SCIN is downregulated in human HCC. In order to determine the expression pattern of SCIN in HCC tissues, the present study detected mRNA expression in 48 paired HCC tissues and their normal counterparts via RT-qPCR. The results demonstrated that SCIN was downregulated in 39 out of 48 of the paired tissues (Fig. 1A). In order to verify this result, the SCIN protein level was examined in the $12 \mathrm{HCC}$ tissues and the respective non-cancerous tissues. A lower SCIN protein level was consistently observed in 10 out of the 12 paired HCC tissues (Fig. 1B). These results prompted the assessment of the clinical outcome of SCIN downregulation. In order to achieve this, a TMA comprised of the 60 paired HCC tissues and respective normal counterparts was stained using the standard IHC method. While the signal for SCIN was considered strong in peri-tumor normal hepatocytes, less signals were observed in the HCC tissues (Fig. 1C). The TMA was subsequently analyzed and the signal was reflected via the $\mathrm{H}$-score. The results of the present study demonstrated that SCIN was significantly downregulated in HCC tissues compared with normal tissues (the average $\mathrm{H}$-score for the normal tissue group was 7.4 vs. 4.2 for the HCC group; $\mathrm{P}<0.0001$; Fig. 1D). Thus, the results of the present study suggest that SCIN is downregulated in HCC.

SCIN expression is associated with clinicopathological features and predicts prognosis. Next, the association between SCIN expression and clinical features was analyzed using the TMA data. Patients were divided into two groups according to the H-score for their HCC tissues (patients with $\mathrm{H}$-scores $<6$ were categorized into the low SCIN expression group, $\mathrm{n}=39$; patients with $\mathrm{H}$-score $\geq 6$ were categorized into the high SCIN expression group, $n=21$ ). The results of the present 
A

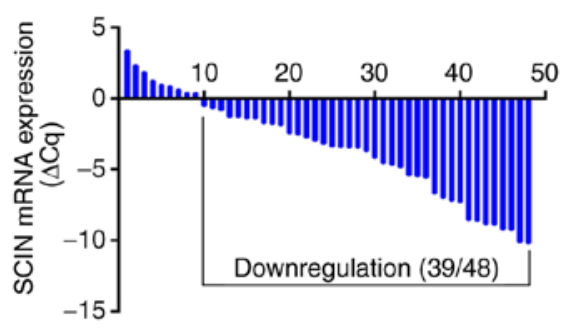

B
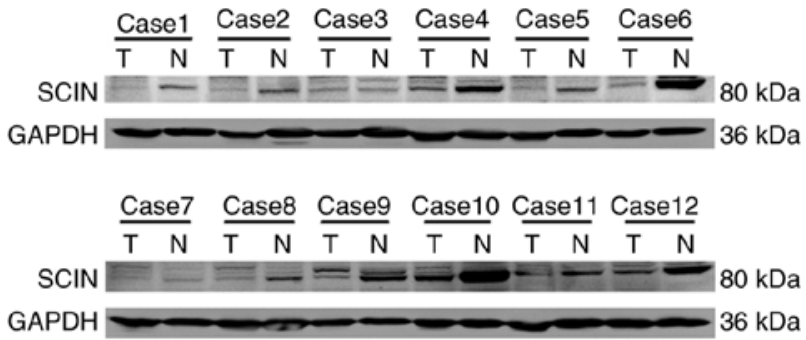

C
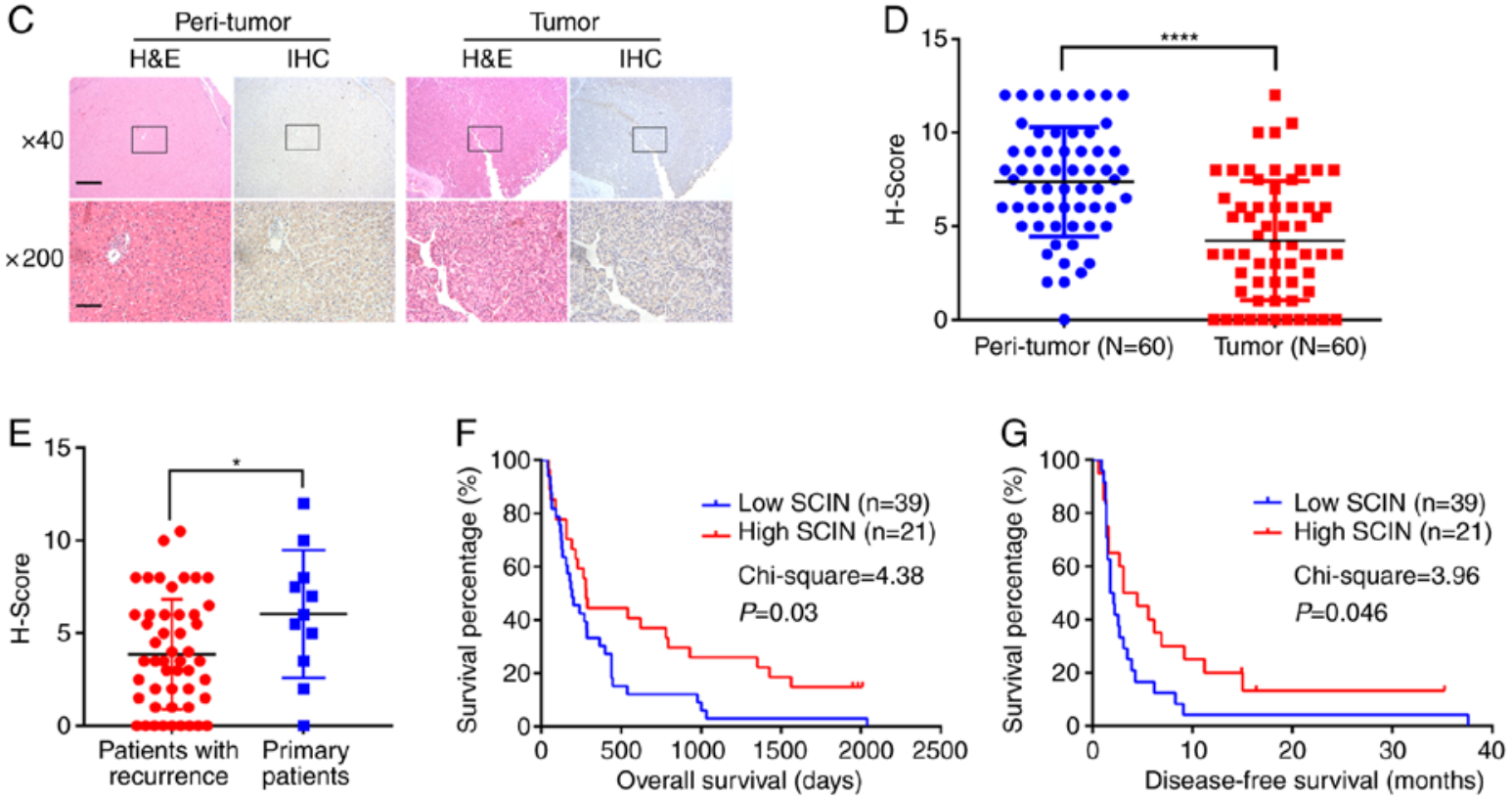

Figure 1. SCIN is downregulated in HCC and low expression predicts poor survival. (A) Reverse transcription-quantitative PCR analysis of SCIN mRNA expression in the 48 paired pancreatic ductal adenocarcinoma tumor tissues and normal tissues. (B) Western blot analysis of SCIN protein expression in the 12 paired HCC tissues. (C) Representative images of H\&E and IHC staining of SCIN in TMA. Magnification, x40 (scale bar, $500 \mu$ m) and x200 (scale bar, $100 \mu \mathrm{m}$ ). (D and E) Dot plot H-score of SCIN indicated staining signal in TMA containing the 60 paired HCC and normal tissues. (F) KM overall survival analysis of patients in SCIN low expression and SCIN high expression groups. (G) KM disease-free survival analysis of patients in SCIN low expression and SCIN high expression groups. ${ }^{*} \mathrm{P}<0.05$ and ${ }^{* * * *} \mathrm{P}<0.0001$. SCIN, scinderin; HCC, hepatocellular carcinoma; H\&E, hematoxylin and eosin; IHC, immunohistochemistry; TMA, tissue microarray; KM, Kaplan-Meier; T, tumor; N, normal.

study demonstrated that SCIN expression was significantly associated with tumor size (Table I); however, no significant associations were observed between SCIN expression and lymph node (LN) metastases, cirrhosis, age or microvascular invasion (MVI). The association between SCIN expression and the survival and recurrence status for the 60 patients was subsequently analyzed. The results revealed that SCIN expression was significantly decreased in patients with recurrence compared with patients without recurrence (Fig. 1E). Furthermore, the low SCIN expression group demonstrated a shorter overall survival (OS) time $\left(\chi^{2}=4.38 ; \mathrm{P}=0.03\right.$; Fig. $\left.1 \mathrm{~F}\right)$, as well as a shorter disease-free survival (DFS) time $\left(\chi^{2}=3.96\right.$; $\mathrm{P}=0.046$; Fig. 1G) compared with the high SCIN expression group. Detailed analysis in the present study demonstrated that the 1- and 3-year recurrence rates of the high SCIN expression group [57.1\% (12/21) and $71.4 \%(15 / 21)$, respectively] were significantly lower than those of the low SCIN expression group [74.4\% (29/39) and 84.6\% (33/39), respectively]. Furthermore, the 1- and 3-year OS times of the high SCIN expression group [66.7\% (14/21) and 28.6\% (6/21), respectively] were significantly longer than those of the low SCIN expression group $[38.5 \%$
$(15 / 39)$ and $10.3 \%$ (4/39), respectively; Table II]. In order to further assess whether SCIN expression could serve as an independent risk factor for both OS and DFS in HCC, the Cox proportional hazards model was implemented in the present study. Factors included in this model were as follows: Sex, age, tumor size, serum $\alpha$-fetoprotein (AFP), serum HBsAg, serum CA199, encapsulation, liver cirrhosis, tumor number, SCIN expression, LN metastases and MVI. SCIN and MVI were found to be associated using Cox analysis (Tables III and IV). The results indicate that SCIN expression has the ability to serve as an independent prognostic factor, affecting both DFS and OS in HCC. SCIN expression has a notable association with the good prognosis of patients with HCC and thus could serve as an independent risk factor for survival.

SCIN suppresses HCC cell proliferation. The present study utilized gene manipulation mediated by lentivirus in order to assess the function of SCIN in HCC cells. SCIN expression in six HCC cell lines was determined (Fig. 2A). In order to investigate the role of SCIN in HCC cells, the present study overexpressed SCIN in SK-HEP-1 cells, which exhibited low 
Table I. Association between SCIN expression and clinicopathological features of patients with hepatocellular carcinoma.

\begin{tabular}{|c|c|c|c|c|}
\hline \multirow[b]{2}{*}{ Characteristic } & \multirow[b]{2}{*}{ Patients, $\mathrm{n}$} & \multicolumn{2}{|c|}{ SCIN expression, $\mathrm{n}$} & \multirow[b]{2}{*}{ P-value } \\
\hline & & Low $(n=39)$ & High $(n=21)$ & \\
\hline Age, years & & & & 0.679 \\
\hline$<50$ & 42 & 28 & 14 & \\
\hline$\geq 50$ & 18 & 11 & 7 & \\
\hline Tumor size, $\mathrm{cm}$ & & & & $0.028^{\mathrm{a}}$ \\
\hline$<5$ & 23 & 11 & 12 & \\
\hline$\geq 5$ & 37 & 28 & 9 & \\
\hline Liver cirrhosis & & & & 0.740 \\
\hline Presence & 36 & 24 & 12 & \\
\hline Absence & 24 & 15 & 9 & \\
\hline Lymph node metastases & & & & 0.717 \\
\hline Positive & 10 & 6 & 4 & \\
\hline Negative & 50 & 33 & 17 & \\
\hline Microvascular invasion & & & & 0.251 \\
\hline Positive & 20 & 15 & 5 & \\
\hline Negative & 40 & 24 & 16 & \\
\hline
\end{tabular}

${ }^{\mathrm{a}} \mathrm{P}<0.05 . \mathrm{SCIN}$, scinderin.

Table II. 1-year and 3-year recurrence rates and overall survival rates of patients.

\begin{tabular}{|c|c|c|c|c|c|}
\hline \multirow[b]{2}{*}{ SCIN expression } & \multirow[b]{2}{*}{ Patients, $\mathrm{n}$} & \multicolumn{2}{|c|}{ Recurrence rates, $\%$ (n) } & \multicolumn{2}{|c|}{ Overall survival rates, $\%$ (n) } \\
\hline & & 1-year & 3-year & 1-year & 3-year \\
\hline High & 21 & $57.1 \%(12)$ & $71.4 \%(15)$ & $66.7 \%(14)$ & $28.6 \%(6)$ \\
\hline Low & 39 & $74.4 \%(29)$ & $84.6 \%(33)$ & $38.5 \%(15)$ & $10.3 \%(4)$ \\
\hline
\end{tabular}

SCIN, scinderin.

endogenous SCIN levels, and knocked down SCIN expression in YY-8103 cells, which manifested high endogenous SCIN levels (Fig. 2B). Considering that SCIN expression was inversely associated with tumor size clinically, the present study subsequently examined the proliferative ability of these cells via MTT assays. The results of the present study demonstrated that while overexpression of SCIN in SK-HEP-1 cells inhibited cell proliferation, SCIN knockdown in YY-8103 cells promoted cell proliferation (Fig. 2C). Consistent with these results, the present study demonstrated that while SCIN overexpression in SK-HEP-1 cells decreased the colony-formation ability of these cells, its inhibition increased the colony-formation ability in YY-8103 cells (Fig. 2D). Overall, the results of the present study suggest that SCIN suppresses HCC cell proliferation in vitro.

In order to further investigate the role of SCIN, in vivo experiments were performed in the present study. YY-8103 shSCIN and shcon cells were injected subcutaneously into the left and right flanks of the six nude mice. SCIN-knockdown tumors grew faster than those in the control group (Fig. 3A).
After 4 weeks, the mice were sacrificed and all the tumors were weighed. The results demonstrated that the tumors in the shSCIN group were significantly heavier $(\mathrm{P}<0.001$; Fig. $3 \mathrm{~B})$ and larger (Fig. 3C) than those in the control group. Consistently, SCIN overexpression in SK-HEP-1 cells inhibited the in vivo tumor formation ability (Fig. 3D and E). The tumor lengths and volumes for all groups are presented in Table V. Overall, the results of the present study suggest that SCIN promotes tumorigenesis in vivo.

SCIN expression arrests cells in the $S / G_{2}$ phase, and downregulates pSTAT3 and cyclin A1. The present study performed FACs in order to analyze the cell cycle distribution in SCIN-manipulated cells, to better understand how SCIN inhibits cell proliferation. The results of the present study demonstrated that SCIN overexpression in SK-HEP-1 cells markedly increased the number of cells in the $S$ phase (from 25.6-42.0\%) and decreased the number of cells in the $\mathrm{G}_{2}$ phase (from 17.5-5.2\%) (Fig. 4A), indicating that SCIN expression results in cell cycle arrest in the $S / G_{2}$ phase. Similarly, 
Table III. Cox regression analysis of OS in patients with hepatocellular carcinoma.

\begin{tabular}{|c|c|c|c|c|}
\hline \multirow[b]{3}{*}{ Variable } & \multicolumn{4}{|c|}{ OS } \\
\hline & \multicolumn{2}{|c|}{ Univariate } & \multicolumn{2}{|c|}{ Multivariate } \\
\hline & HR $(95 \%$ CI $)$ & P-value & HR $(95 \%$ CI $)$ & P-value \\
\hline Sex & $0.706(0.274-1.821)$ & 0.472 & & \\
\hline Age & $0.656(0.307-1.398)$ & 0.275 & & \\
\hline Tumor size & $1.194(0.612-2.330)$ & 0.603 & & \\
\hline Serum AFP & $1.328(0.515-3.426)$ & 0.557 & & \\
\hline Serum HBsAg & $1.911(0.673-5.429)$ & 0.224 & & \\
\hline Serum CA19-9 & $1.791(0.732-4.382)$ & 0.202 & & \\
\hline Encapsulation & $1.105(0.530-2.303)$ & 0.782 & & \\
\hline Liver cirrhosis & $2.187(1.073-4.461)$ & $0.031^{\mathrm{a}}$ & $1.948(0.887-4.278)$ & 0.097 \\
\hline Tumor number & $2.401(1.021-5.647)$ & $0.045^{\mathrm{a}}$ & $1.298(0.365-4.615)$ & 0.687 \\
\hline SCIN & $0.424(0.203-0.887)$ & $0.023^{\mathrm{a}}$ & $0.384(0.175-0.842)$ & $0.017^{\mathrm{a}}$ \\
\hline Lymph node metastases & $3.342(1.403-7.957)$ & $0.006^{\mathrm{a}}$ & $1.793(0.492-6.539)$ & 0.377 \\
\hline Microvascular invasion & 4.357 (1.966-9.654) & $<0.001^{\mathrm{a}}$ & $2.668(1.122-6.344)$ & $0.026^{\mathrm{a}}$ \\
\hline
\end{tabular}

${ }^{\mathrm{a}} \mathrm{P}<0.05$. OS, overall survival; serum AFP, serum $\alpha$-fetoprotein; CA19-9, carbohydrate antigen 19-9; HBsAg, hepatitis B surface antigen; SCIN, scinderin; HR, hazard ratio; CI, confidence interval.

Table IV. Cox regression analysis of DFS in patients with hepatocellular carcinoma.

\begin{tabular}{|c|c|c|c|c|}
\hline \multirow[b]{3}{*}{ Variable } & \multicolumn{4}{|c|}{ DFS } \\
\hline & \multicolumn{2}{|c|}{ Univariate } & \multicolumn{2}{|c|}{ Multivariate } \\
\hline & $\operatorname{HR}(95 \% \mathrm{CI})$ & P-value & $\operatorname{HR}(95 \% \mathrm{CI})$ & P-value \\
\hline Sex & $0.682(0.304-1.533)$ & 0.355 & & \\
\hline Age & $0.613(0.322-1.167)$ & 0.136 & & \\
\hline Tumor size & $1.426(0.786-2.585)$ & 0.243 & & \\
\hline Serum AFP & $0.792(0.370-1.695)$ & 0.548 & & \\
\hline Serum HBsAg & $2.221(0.936-5.271)$ & 0.070 & & \\
\hline Serum CA19-9 & $1.459(0.647-3.286)$ & 0.362 & & \\
\hline Encapsulation & $1.031(0.561-1.896)$ & 0.921 & & \\
\hline Liver cirrhosis & $1.816(1.009-3.266)$ & $0.046^{\mathrm{a}}$ & $1.634(0.893-2.989)$ & 0.111 \\
\hline Tumor number & $1.747(0.879-3.473)$ & 0.112 & & \\
\hline SCIN & $0.465(0.251-0.862)$ & $0.015^{\mathrm{a}}$ & $0.484(0.259-0.904)$ & $0.023^{\mathrm{a}}$ \\
\hline Lymph node metastases & $1.892(0.890-4.022)$ & 0.098 & & \\
\hline Microvascular invasion & $2.950(1.541-5.646)$ & $0.001^{\mathrm{a}}$ & $2.605(1.339-5.071)$ & $0.005^{\mathrm{a}}$ \\
\hline
\end{tabular}

${ }^{\mathrm{a} P}<0.05$. DFS, disease-free survival; serum AFP, serum $\alpha$-fetoprotein; CA19-9, carbohydrate antigen 19-9; HBs Ag, hepatitis B surface antigen; SCIN, scinderin; HR, hazard ratio; CI, confidence interval.

SCIN-knockdown in YY-8103 cells demonstrated a decrease in the number of cells in the $\mathrm{S}$ phase and an increase in the number of cells in the $\mathrm{G}_{2}$ phase (Fig. 4B). Thus, the results of the present study indicate that cells are arrested in the $S / G_{2}$ transition following SCIN expression.

As the cell cycle is regulated by cyclins (21), the present study examined whether the expression of cyclins was altered following SCIN overexpression or knockdown. The results of the present study demonstrated that while SCIN overexpression downregulated cyclin A1, the protein level of cyclin A1 increased following SCIN knockdown (Fig. 4C). As SCIN is associated with F-actin, which is localized in the cell periphery $(8,10)$, there may be oncogenic signaling pathways influenced by SCIN, which could regulate cyclin A1 expression. In order to investigate this, the present study assessed changes in some oncogenic molecules and demonstrated that 
A

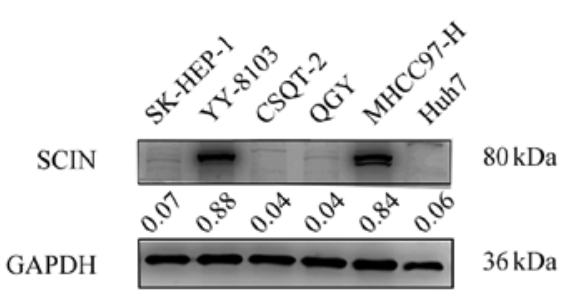

C
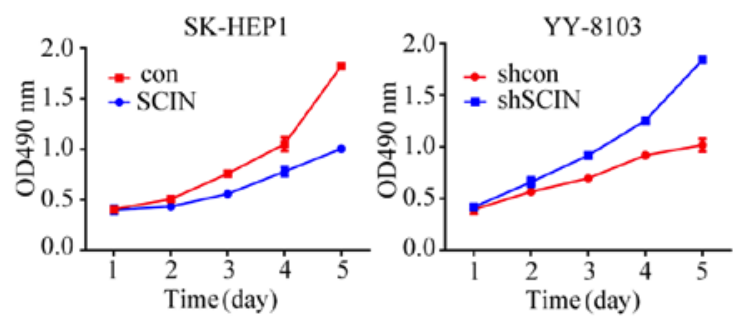

B

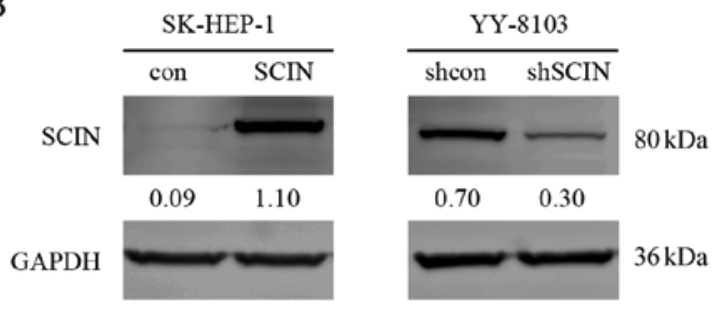

D

SK-HEP-1
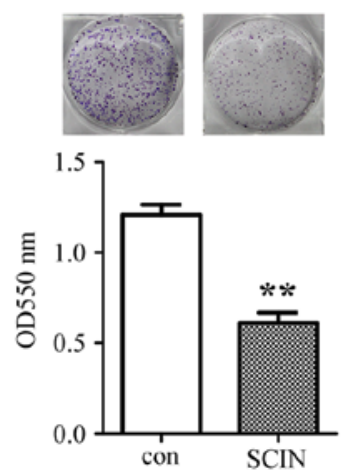

YY-8103
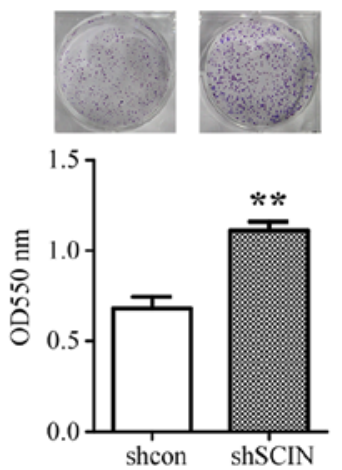

Figure 2. SCIN inhibits HCC cell proliferation in vitro. (A) Western blot analysis of SCIN protein expression in six HCC cell lines. (B) Western blot analysis demonstrating SCIN overexpression (left) and SCIN-knockdown (right) efficiency in the indicated cell lines. (C) Cell growth rate curves demonstrating absorbance at the indicated days. (D) Representative images (upper) and bar plot (lower) of the colony formation assays. ${ }^{* *} \mathrm{P}<0.01$, SK-HEP-1 con vs. SK-HEP-1 SCIN and YY-8103-shcon vs. YY-8103-shSCIN. SCIN, scinderin; HCC, hepatocellular carcinoma; con, control; shSCIN, short hairpin scinderin; shcon, short hairpin control; OD, optical density.

A

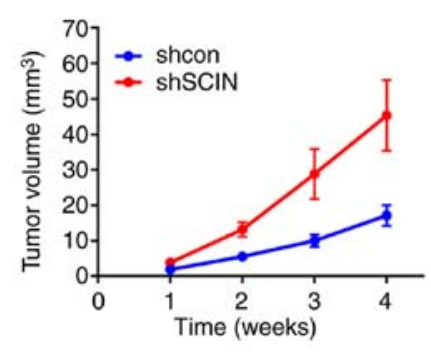

B

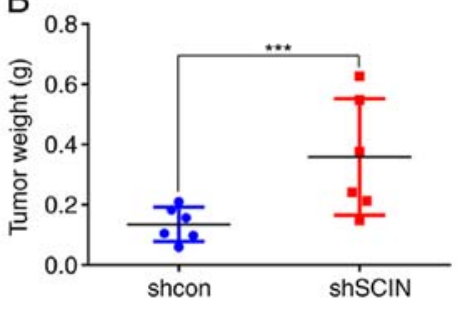

C

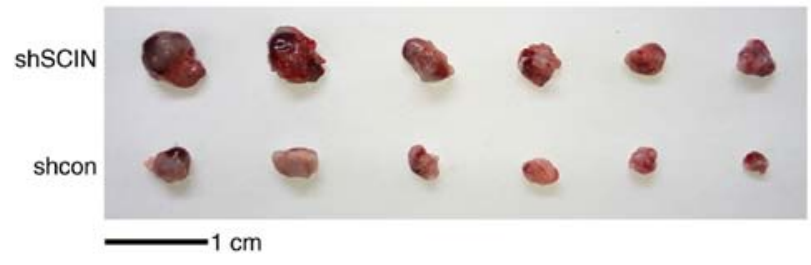

D

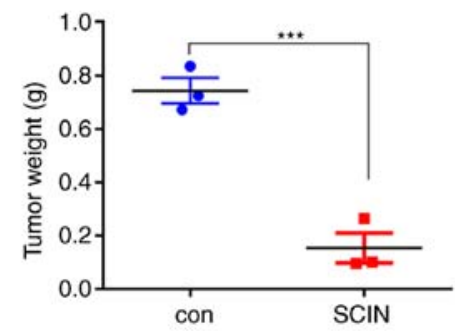

$\mathrm{E}$

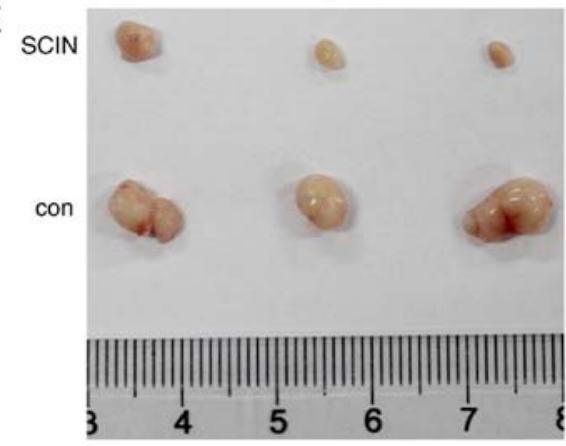

Figure 3. SCIN inhibits HCC cell proliferation in vivo. (A) Line chart of tumor volume in YY-8103 cells. (B) Dot plot of tumor weight in YY-8103 cells. (C) Images of tumors isolated from the nude mice in YY-8103 cells. (D) Dot plot of tumor weight in SK-HEP-1 cells. (E) Images of tumors isolated from the nude mice in SK-HEP-1 cells. ${ }^{* * *} \mathrm{P}<0.001$. SCIN, scinderin; HCC, hepatocellular carcinoma; shcon, short hairpin control; shSCIN, short hairpin scinderin; con, control. 
A
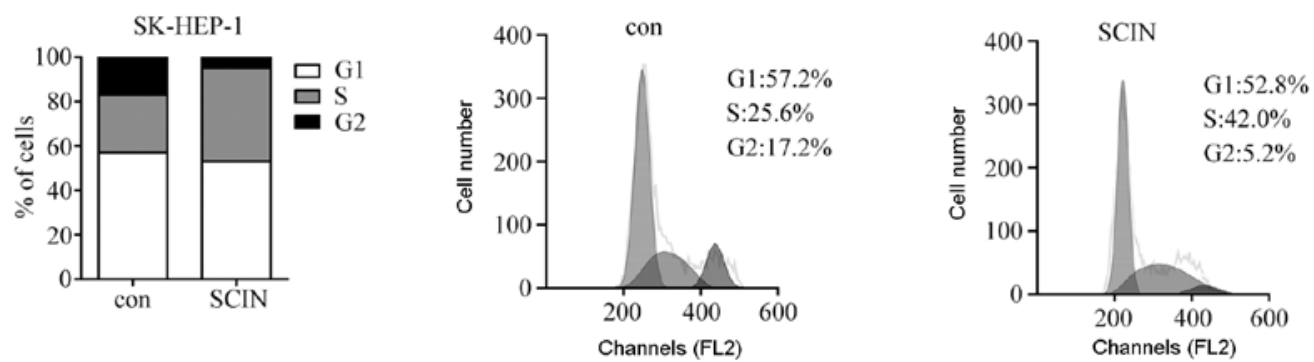

$\mathrm{B}$
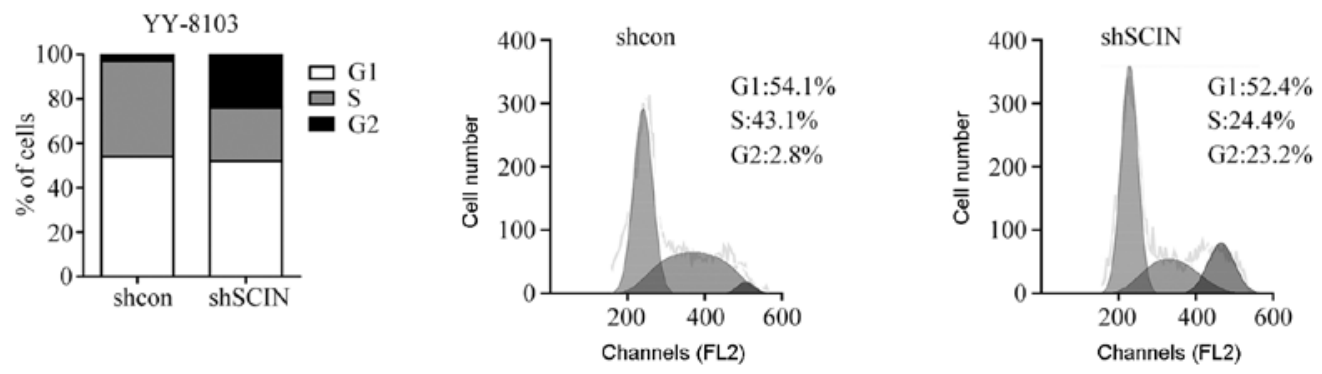

$\mathrm{C}$
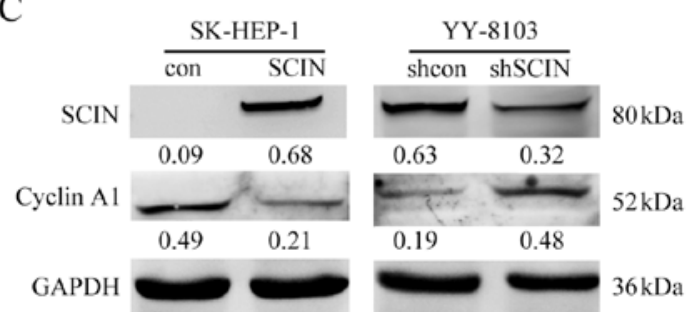

E

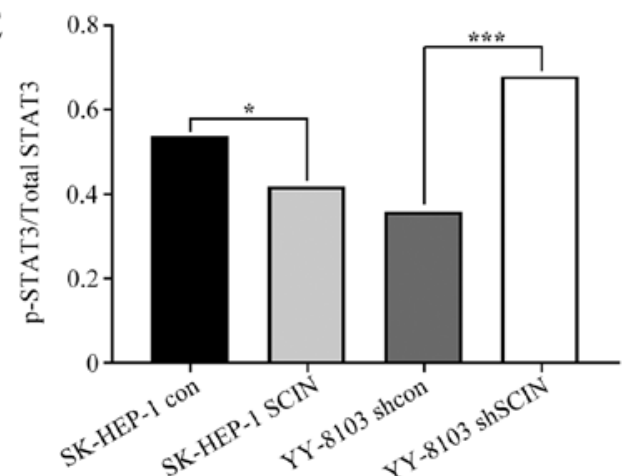

D
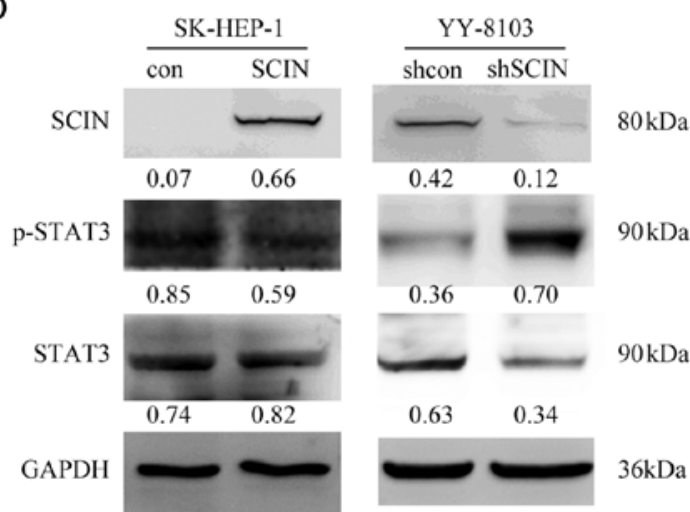

Figure 4. SCIN promotes cell cycle arrest at the $\mathrm{S} / \mathrm{G}_{2}$ phase and regulates oncogenic proteins. (A) Bar plot of cell cycle distribution of SK-HEP-1 cells and (B) YY-8103 cells. (C) Western blot analysis of cyclin A1 in the indicated cell lines. (D) Western blot analysis of p-STAT3 in the indicated cell lines. (E) Ratio of p-STAT3 and total STAT3 in SK-HEP-1 and YY-8103 cells. ${ }^{*} \mathrm{P}<0.05$ and ${ }^{* * *} \mathrm{P}<0.001$. SCIN, scinderin; p-STAT3, phosphorylated-signal transducer and activator of transcription 3; con, control; sh, short hairpin.

the phosphorylation of signal transducer and activator of transcription 3 (STAT3) was inversely associated with cellular SCIN levels (Fig. 4D and E). These observations indicate that cyclin A1 and phospho-STAT3 are negatively regulated by SCIN.

\section{Discussion}

SCIN has been reported to possess an oncogenic role in several types of solid tumor by promoting tumor cell proliferation and invasion $(10,12)$. In certain types of leukemia, SCIN has been demonstrated to be downregulated and its expression associated with the induction of cell differentiation $(8,14)$. Hasmim et al (15) demonstrated the weak expression of SCIN in 9 out of 83 patients with head and neck cancer, indicating that the role of SCIN may vary within different types of tumor. This paradoxical function of SCIN in different types of cancer may be attributed to different genetic backgrounds and distinct tumor milieu (22). However, the present study demonstrated that SCIN was tumor suppressive in HCC. The results of the present study demonstrated that SCIN was downregulated in samples derived from patients with HCC, and notably, the low expression of SCIN in resected HCC tissues predicted poor prognosis in postoperative patients.

SCIN expression status, combined with clinicopathological features and other biomarkers of HCC may be useful for the development of individualized treatment in patients with HCC. However, further investigations in other cohorts are 
Table V. Volumes and lengths of each tumor.

\begin{tabular}{|c|c|c|c|}
\hline $\begin{array}{l}\text { Tumor } \\
\text { number }\end{array}$ & $\begin{array}{l}\text { Length }(\mathrm{A}), \\
\mathrm{mm}\end{array}$ & $\begin{array}{l}\text { Length }(\mathrm{B}) \text {, } \\
\text { mm }\end{array}$ & $\begin{array}{c}\text { avolume, } \\
\mathrm{mm}^{3}\end{array}$ \\
\hline \multicolumn{4}{|c|}{ YY-8103 shSCIN } \\
\hline 1 & 6.5 & 4.9 & 78.03 \\
\hline 2 & 6.0 & 4.8 & 69.12 \\
\hline 3 & 5.3 & 4.2 & 46.75 \\
\hline 4 & 4.0 & 3.9 & 30.42 \\
\hline 5 & 3.9 & 3.1 & 18.74 \\
\hline 6 & 3.9 & 3.7 & 26.70 \\
\hline \multicolumn{4}{|c|}{ YY-8103 shcon } \\
\hline 7 & 3.9 & 3.6 & 25.27 \\
\hline 8 & 4.0 & 3.4 & 23.12 \\
\hline 9 & 3.8 & 2.5 & 11.88 \\
\hline 10 & 3.9 & 3.2 & 19.97 \\
\hline 11 & 3.0 & 3.0 & 13.50 \\
\hline 12 & 2.7 & 2.4 & 7.78 \\
\hline \multicolumn{4}{|c|}{ SK-HEP-1 SCIN } \\
\hline 13 & 4.4 & 3.9 & 33.46 \\
\hline 14 & 3.5 & 2.7 & 12.76 \\
\hline 15 & 3.1 & 2.8 & 12.15 \\
\hline \multicolumn{4}{|c|}{ SK-HEP-1 con } \\
\hline 16 & 7.5 & 4.9 & 90.04 \\
\hline 17 & 6.1 & 5.3 & 85.67 \\
\hline 18 & 9.0 & 4.8 & 103.68 \\
\hline
\end{tabular}

${ }^{\mathrm{a}}$ Volume $=\mathrm{A} \mathrm{x} \mathrm{B}^{2} / 2$. shSCIN, short hairpin scinderin; shcon, short hairpin control.

required in order to verify these hypotheses. As the number of cases in the present study was limited, the association between SCIN expression and HCC requires further evaluation. Longer follow-up studies are required in order to further investigate the significance of SCIN in HCC.

$\mathrm{HCC}$ is one of the many types of cancer closely associated with inflammation and infection (23). One feature of HCC development is continuous hepatocyte death followed by inflammatory infiltration and liver regeneration (24). The IL6/STAT3 signaling pathway is a notable pathway involved in the death-inflammation-regeneration process (25). Universal STAT3 activation in HCC has previously been reported in a number of studies $(26,27)$, whereby patients exhibiting STAT3 activation tend to have a poor prognosis. The present study demonstrated that SCIN could negatively regulate the activation of STAT3, which underlined the regulation of this molecule. Although the present study failed to clarify the molecular mechanisms by which SCIN deregulates STAT3 activation, it was hypothesized that SCIN may modulate some aspects of upstream receptors or Janus kinases considering that SCIN is associated with F-actin, which in turn is closely associated with membrane receptors in space $(28,29)$. Thus, further studies are required in order to demonstrate STAT3 regulation via SCIN.

The results of the present study demonstrated that SCIN has the ability to regulate cyclin A1 protein levels in HCC cells. In the cell cycle process, cyclin A1 is present at very low levels during the $\mathrm{G}_{0}$ phase; it increases throughout the progression of the cell cycle and reaches peak levels in the $\mathrm{S}$ phase and during the $\mathrm{G}_{2} / \mathrm{M}$ phase (30). The results of the present study demonstrated that SCIN has the ability to induce cell cycle arrest, which increased the percentage of cells in the $S$ phase and inhibited entry into the $G_{2}$ phase. This phenotype may be explained by the influence of SCIN on cyclin A1 expression; however, the association between SCIN and cyclin A1 may be indirect as SCIN is not a transcription factor. Thus, some signaling pathways may mediate cyclin A1 expression via SCIN.

Overall, the present study demonstrated that SCIN expression was downregulated in HCC and that SCIN functioned to suppress the proliferation of HCC cells, which may be mediated by cyclin A1 and STAT3, suggesting that SCIN may be a tumor suppressor gene in HCC. Furthermore, SCIN may serve as a valuable prognostic marker, as well as a potential therapeutic target for HCC.

\section{Acknowledgements}

The authors would like to thank Dr Liu Shanrong (Department of Clinical Laboratory, Changhai Hospital, Shanghai, China) for providing advice on experimental ideas.

\section{Funding}

The present study was funded by The National Key Basic Research Program 973 Project (grant no. 2015CB554000), The Project of Shanghai Shenkang Hospital Development Center (grant no. SHDC12015106), The Key Project of the Natural Science Foundation of China (grant no. 81730097), The Science Fund for Creative Research Groups (grant no. 81521091) and The Chang Jiang Scholars Program (year 2013) of the Ministry of Education of the People's Republic of China.

\section{Availability of data and materials}

The datasets used and/or analyzed during the current study are available from the corresponding author on reasonable request.

\section{Authors' contributions}

BZ, TWC and YBJ made substantial contributions to the conception and design of the study. XBW and CDL were responsible for the analysis and interpretation of data. DX and JJL participated in the design of the study and experimentation, and were involved in drafting the manuscript. SQC participated in the experiment design and the subject establishment of this article, as well as critically revising the manuscript approving the submitted version. All authors read and approved the final manuscript.

\section{Ethics approval and consent to participate}

The human and animal experiments performed in the present study were approved by the Medical Ethics Committee of Eastern Hepatobiliary Surgery Hospital (Shanghai, China). All patients provided written informed consent prior to the study. 


\section{Patient consent for publication}

Not applicable.

\section{Competing interests}

The authors declare that they have no competing interests.

\section{References}

1. Siegel RL, Miller KD and Jemal A: Cancer statistics, 2019. CA Cancer J Clin 69: 7-34, 2019.

2. Orcutt ST and Anaya DA: Liver resection and surgical strategies for management of primary liver cancer. Cancer Control 25 $1073274817744621,2018$.

3. Zhang Y, Liu S, Wang X, Zhang Z, Jing X, Zhang P and Xie Z: Prevention of local liver cancer recurrence after surgery using multilayered cisplatin-loaded polylactide electrospun nanofbers. Chin J Polym Sci 32: 1111-1118, 2014.

4. Zhang XP, Chen ZH, Zhou TF, Li LQ, Chen MS, Wen TF, Shi J, Guo WX, Wu MC, Lau WY, et al: A nomogram to predict early postoperative recurrence of hepatocellular carcinoma with portal vein tumour thrombus after R0 liver resection: A large-scale, multicenter study. Eur J Surg Oncol 45: 1644-1651, 2019.

5. Rodríguez Del Castillo A, Vitale ML, Tchakarov L and TrifaróJM: Human platelets contain scinderin, a $\mathrm{Ca}(2+)$-dependent actin filament-severing protein. Thromb Haemost 67: 248-251, 1992.

6. Dumitrescu Pene T, Rosé SD, Lejen T, Marcu MG and Trifaró JM: Expression of various scinderin domains in chromaffin cells indicates that this protein acts as a molecular switch in the control of actin filament dynamics and exocytosis. J Neurochem 92: 780-789, 2005.

7. Trifaro JM, Gasman S and Gutierrez LM: Cytoskeletal control of vesicle transport and exocytosis in chromaffin cells. Acta Physiol (Oxf) 192: 165-172, 2008.

8. Zunino R, Li Q, Rosé SD, Romero-Benítez MM, Lejen T, Brandan NC and Trifaró JM: Expression of scinderin in megakaryoblastic leukemia cells induces differentiation, maturation, and apoptosis with release of plateletlike particles and inhibits proliferation and tumorigenesis. Blood 98: 2210-2219, 2001.

9. Jiang H, Wang Y, Viniegra A, Sima C, McCulloch CA and Glogauer M: Adseverin plays a role in osteoclast differentiation and periodontal disease-mediated bone loss. FASEB J 29: 2281-2291, 2015

10. Wang D, Sun SQ, Yu YH, Wu WZ, Yang SL and Tan JM: Suppression of SCIN inhibits human prostate cancer cell proliferation and induces G0/G1 phase arrest. Int J Oncol 44: 161-166, 2014.

11. Liu H, Shi D, Liu T, Yu Z and Zhou C: Lentivirus-mediated silencing of SCIN inhibits proliferation of human lung carcinoma cells. Gene 554: 32-39, 2015.

12. Liu JJ, Liu JY, Chen J, Wu YX, Yan P, Ji CD, Wang YX, Xiang DF, Zhang $\mathrm{X}$, Zhang $\mathrm{P}$, et al: Scinderin promotes the invasion and metastasis of gastric cancer cells and predicts the outcome of patients. Cancer Lett 376: 110-117, 2016.

13. Chen XM, Guo JM, Chen P, Mao LG, Feng WY, Le DH and Li KQ: Suppression of scinderin modulates epithelial-mesenchymal transition markers in highly metastatic gastric cancer cell line SGC-7901. Mol Med Rep 10: 2327-2333, 2014.

14. Zhang ZH, Zhang W, Zhou JD, Zhang TJ, Ma JC, Xu ZJ, Lian XY, Wu DH, Wen XM, Deng ZQ, et al: Decreased SCIN expression, associated with promoter methylation, is a valuable predictor for prognosis in acute myeloid leukemia. Mol Carcinog 57: 735-744, 2018 .
15. Hasmim M, Badoual C, Vielh P, Drusch F, Marty V, Laplanche A, de Oliveira Diniz M, Roussel H, De Guillebon E, Oudard S, et al: Expression of EPHRIN-A1, SCINDERIN and MHC class I molecules in head and neck cancers and relationship with the prognostic value of intratumoral $\mathrm{CD} 8^{+} \mathrm{T}$ cells. BMC Cancer 13: 592, 2013

16. European Association for the Study of the Liver. Electronic address: easloffice@easloffice.eu European Association for the Study of the Liver: EASL clinical practice guidelines: Management of hepatocellular carcinoma. J Hepatol 69: 182-236, 2018.

17. Wang T, Hu HS, Feng YX, Shi J, Li N, Guo WX, Xue J, Xie D, Liu SR, Wu MC and Cheng SQ: Characterisation of a novel cell line (CSQT-2) with high metastatic activity derived from portal vein tumour thrombus of hepatocellular carcinoma. $\mathrm{Br}$ J Cancer 102: 1618-1626, 2010.

18. Li JJ, Liu DP, Liu GT and Xie D: EphrinA5 acts as a tumor suppressor in glioma by negative regulation of epidermal growth factor receptor. Oncogene 28: 1759-1768, 2009.

19. Livak KJ and Schmittgen TD: Analysis of relative gene expression data using real-time quantitative PCR and the 2(-Delta Delta C(T)) method. Methods 25: 402-408, 2001.

20. Deng YZ, Chen PP, Wang Y, Yin D, Koeffler HP, Li B, Tong XJ and Xie D: Connective tissue growth factor is overexpressed in esophageal squamous cell carcinoma and promotes tumorigenicity through beta-catenin-T-cell factor/Lef signaling. J Biol Chem 282: 36571-36581, 2007.

21. Ehedego H, Mohs A, Jansen B, Hiththetiya K, Sicinski P, Liedtke $\mathrm{C}$ and Trautwein C: Loss of Cyclin E1 attenuates hepatitis and hepatocarcinogenesis in a mouse model of chronic liver injury. Oncogene 37: 3329-3339, 2018.

22. Mohamed E, Al-Khami AA and Rodriguez PC: The cellular metabolic landscape in the tumor milieu regulates the activity of myeloid infiltrates. Cell Mol Immunol 15: 421-427, 2018.

23. Nguyen VT, Law MG and Dore GJ: Hepatitis B-related hepatocellular carcinoma: Epidemiological characteristics and disease burden. J Viral Hepat 16: 453-463, 2009.

24. Block TM, Mehta AS, Fimmel CJ and Jordan R: Molecular viral oncology of hepatocellular carcinoma. Oncogene 22: 5093-5107, 2003.

25. Ye X, Wu H, Sheng L, Liu YX, Ye F, Wang M, Zhou H, Su Y and Zhang XK: Oncogenic potential of truncated RXR $\alpha$ during colitis-associated colorectal tumorigenesis by promoting IL-6-STAT3 signaling. Nat Commun 10: 1463, 2019.

26. He G, Yu GY, Temkin V, Ogata H, Kuntzen C, Sakurai T, Sieghart W, Peck-Radosavljevic M, Leffert HL and Karin M: Hepatocyte IKK beta/NF-kappa B inhibits tumor promotion and progression by preventing oxidative stress-driven STAT3 activation. Cancer Cell 17: 286-297, 2010.

27. Calvisi DF, Ladu S, Gorden A, Farina M, Conner EA, Lee JS, Factor VM and Thorgeirsson SS: Ubiquitous activation of Ras and Jak/Stat pathways in human HCC. Gastroenterology 130: 1117-1128, 2006

28. Yao RR, Li JH, Zhang R, Chen RX and Wang YH: M2-polarized tumor-associated macrophages facilitated migration and epithelial-mesenchymal transition of HCC cells via the TLR4/STAT3 signaling pathway. World J Surg Oncol 16: 9, 2018.

29. Zakaria S, Helmy MW, Salahuddin A and Omran G: Chemopreventive and antitumor effects of benzyl isothiocynate on HCC models: A possible role of HGF/pAkt/STAT3 axis and VEGF. Biomed Pharmacother 108: 65-75, 2018.

30. Yang R, Müller C, Huynh V, Fung YK, Yee AS and Koeffler HP: Functions of cyclin A1 in the cell cycle and its interactions with transcription factor $\mathrm{E} 2 \mathrm{~F}-1$ and the $\mathrm{Rb}$ family of proteins. Mol Cell Biol 19: 2400-2407, 1999. 